\title{
Cerebral aneurysms associated with Behçet's disease: a case report
}

\author{
S Nakasu, M Kaneko, M Matsuda
}

Abstract

Cerebral aneurysms in Behçet's disease are very rare. The role of vasculitis in the aetiology of these aneurysms has not been clarified. A 57 year old man with Behçet's disease is described, who had a subarachnoid hemorrhage due to rupture of a peripheral middle cerebral artery aneurysm. He underwent a successful aneurysmal clipping. Three years later he had seizures and was found to have a new aneurysm on the contralateral peripheral

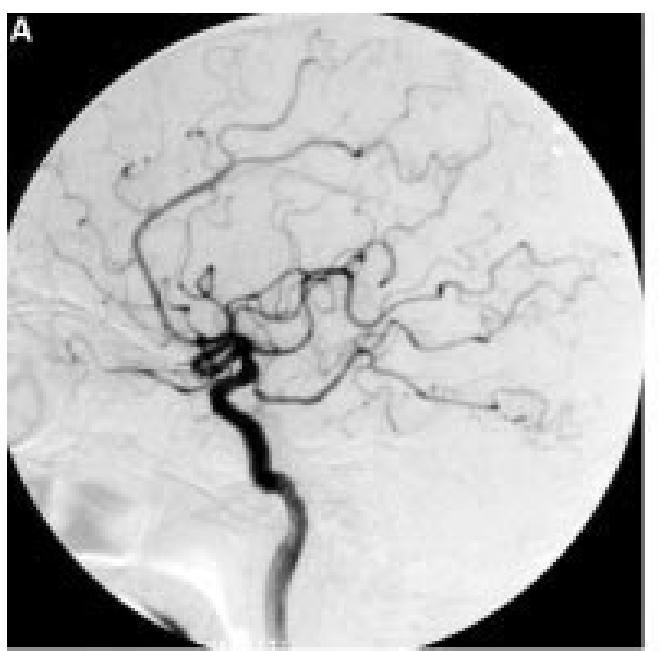

C

Department of

Neurosurgery, Shiga University of Medical Science, Seta, Ohtsu, Shiga 520-2192, Japan S Nakasu

M Kaneko

M Matsuda

Correspondence to:

Dr S Nakasu

snakasu@belle.shiga-med.ac.jp

Received 10 August 2000 and in revised form

6 December 2000

Accepted 13 December 2000 tis

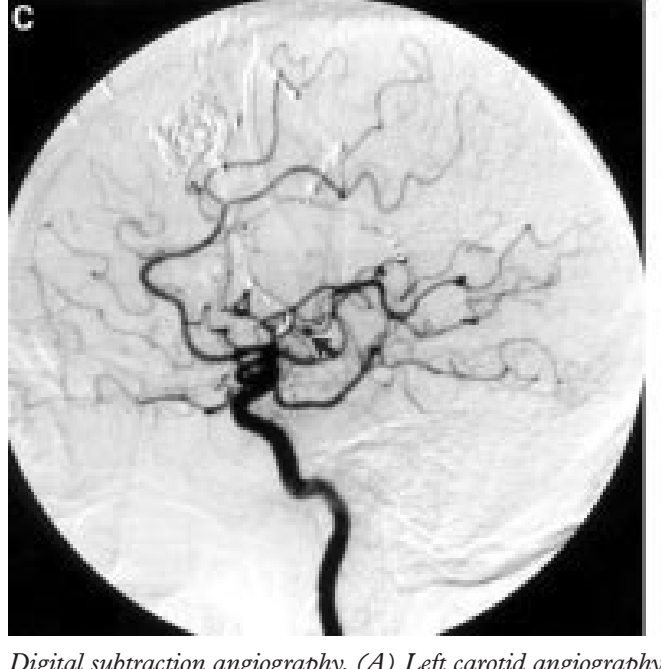
during the steroid therapy showing disappearance of the aneurysm.

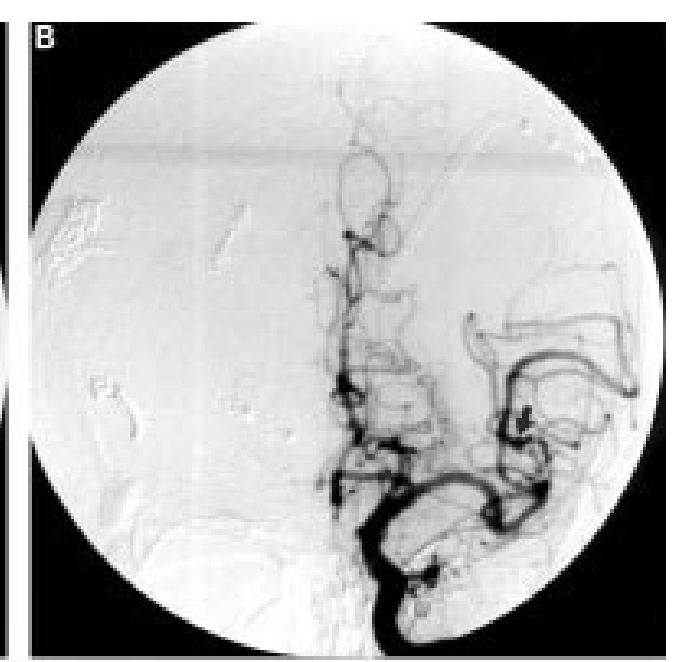

middle cerebral artery as well as some radiological features of vasculitis. After 3 months of steroid therapy, the aneurysm disappeared. Although surgical treatment is the first choice for ruptured aneurysms, steroid therapy may be effective for unruptured small aneurysms in patients with Behçet's disease.

(F Neurol Neurosurg Psychiatry 2001;70:682-684)

Keywords: Behçet's disease; cerebral aneurysm; vasculi-

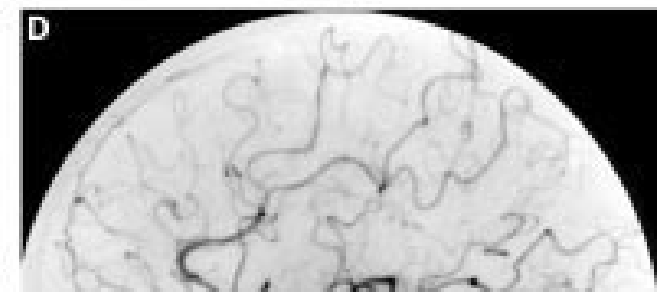

Digital subtraction angiography. (A) Left carotid angiography (CAG) on the first admission showing no aneurysm. (B) Anteroposterior view and $(C)$ lateral view left $C A G$ on the second admission shows a small aneurysm on the peripheral middle cerebral artery (arrows). The peripheral branch shows stenosis and filling delay. (D) Follow up angiography taken 
Table 1 Reported cases of Behçet's disease associated with cerebral aneurysms

\begin{tabular}{|c|c|c|c|}
\hline Authors & Age, sex & Location of aneurysm & Remarks \\
\hline$\overline{\text { Katoh } e t ~ a l^{8}}$ & $29 \mathrm{M}$ & MCA & \\
\hline Buge $e t a l^{9}$ & $43 \mathrm{M}$ & Multiple: ACA, ICA, MCA & Fusiform aneurysms \\
\hline Kerr1 et al $l^{10}$ & $12 \mathrm{M}$ & Multiple: A com, MCA & \\
\hline Tuji et $a l^{11}$ & $62 \mathrm{~F}$ & Multiple, bilateral MCA, ICA, A com & \\
\hline Hassen-Khodja et al ${ }^{12}$ & $43 \mathrm{M}$ & A com & Combined with AVM \\
\hline Ildan $e t a l^{13}$ & $28 \mathrm{M}$ & A com & \\
\hline El Abbadi et al $l^{14}$ & $44 \mathrm{M}$ & Multiple, bilateral MCA & \\
\hline Present case & $57 \mathrm{M}$ & Multiple, bilateral peripheral MCA & Vasculitis \\
\hline
\end{tabular}

$\overline{\mathrm{MCA}=\text { Middle cerebral artery; ICA=internal carotid artery; A com=anterior communicating }}$ artery; $\mathrm{ACA}=$ anteior cerebral artery; $\mathrm{AVM}=$ arteriovenous malformation.

Cerebral vasculitis is a rare cause of cerebral aneurysms. Most patients with this combination have an associated systemic lupus erythematosus. ${ }^{12}$ Very few patients reportedly have isolated CNS angiitis. ${ }^{34}$ Cerebral aneurysms in patients with Behçet's disease are also extremely rare. The role of vasculitis in the aetiology of these aneurysms has not been clarified. We describe a patient with Behçet's disease associated with an unruptured peripheral middle cerebral artery aneurysm that disappeared after steroid therapy.

\section{Case report}

A 57 year old man was admitted to our hospital with a sudden onset of severe headache. He had a history of Behçet's disease since he was 38 years old. At that time, he had exhibited recurrent aphthous stomatitis, skin erythema, and genital ulcers without eye manifestations. He had an aneurysm of the right femoral artery and underwent a replacement of the artery with an artificial vessel. Since then, he had experienced several recurrent episodes, but had been asymptomatic for several years. On admission, he was stuporous without apparent paresis. Brain CT showed a dense subarachnoid haemorrhage in the right sylvian fissure and the insular cistern. Digital subtraction angiography showed a right peripheral middle cerebral artery aneurysm. There were no other aneurysms detected (figure $1 \mathrm{~A}$ ). The aneurysm was clipped successfully. The parent artery looked normal at surgery. During the course of therapy, he transiently exhibited a genital ulcer, but without other signs of Behçet's disease. He underwent a left ventriculoperitoneal shunt for hydrocephalus. He was discharged with moderate memory disturbance. Three years later, he had a generalised tonic seizure and was readmitted to our hospital. Brain CT showed a new low density lesion in the left temporal lobe white matter, which was thought to represent neuroBehçet's disease. Electroencephalography detected spikes in the right frontotemporal region. Digital subtraction angiography showed a small aneurysm on the left peripheral middle cerebral artery (figure $1 \mathrm{~B}, \mathrm{C}$ ). Irregular stenotic lesions were also seen in the bilateral peripheral middle cerebral arteries. Laboratory examination showed an increase in erythrocyte sedimentation rate. The concentrations of C-reactive protein, IgA, and $\mathrm{C} 4$ were also increased. An antineutrophic cytoplasmic antibody and lupus erythematosus test were negative. He remained stuporous for days, but gradually recovered. At that time, he developed aphthous stomatitis. We decided to follow the aneurysm conservatively because it was small and unruptured. He was discharged under steroid therapy $(60 \mathrm{mg}$ prednisolone daily). Three months after initiation of steroid therapy, he was readmitted for an angiographic study. Digital subtraction angiography showed no aneurysm (figure $1 \mathrm{D}$ ), although a mild irregularity of the vessels was still present. The dose of the steroid was gradually tapered over 7 months. After completion of the steroid therapy, follow up digital subtraction angiography showed no aneurysms. However, readministration of the steroid became necessary 3 months later because of recurrent aphthous stomatitis and an increase of C-reactive protein. His psychological manifestations gradually worsened due to progression of neuroBehçet's disease.

\section{Discussion}

Behçet's disease is characterised by specific clinical manifestations including recurrent aphthous stomatitis, genital ulcers, anterior uveitis, and vasculitis. NeuroBehçet's disease occurs, mostly late in the course of the disease, in about $10 \%$ of patients. ${ }^{5}$ The neurological manifestations of the disease are attributed mainly to involvement of the peripheral small arteries or veins, and association of the disease with cerebral aneurysms is very rare. Benamour et $a l^{6}$ and Al-Dalaan et $a l^{7}$ found only one cerebral aneurysm in 316 cases and 119 cases with Behçet's disease, respectively, whereas they did not routinely perform cerebral angiographical studies. Although several authors have described the combination of Behçet's disease with cerebral aneurysms, ${ }^{5-7}$ only eight cases have been reported in detail, including our case (table 1). ${ }^{8-14}$ Most of the aneurysms originated from the common sites of the ordinary saccular aneurysms without radiological features of vasculitis. Five of the eight cases had multiple aneurysms, although this high frequency may have some reporting bias. Buge et al reported multiple fusiform aneurysms. ${ }^{9}$ One aneurysm originated from a feeding artery of an arteriovenous malformation. ${ }^{12}$ Aneurysm formation in vasculoBehçet's disease usually occurs in the systemic major arteries. The histological findings of aneurysms include ruptured internal and external elastic laminae with thickening of the tunica media, and vasculitis of the vasavasorum with perivascular lymphocytic infiltration. ${ }^{5}$ However, in cerebral aneurysms in Behçet's disease, histological examination in two patients showed no features of vasculitis. ${ }^{811}$ Therefore, it is difficult to exclude the possibility of coincidental association in most of the cases. However, the high frequency of multiple aneurysms in these patients may support the likelihood that Behçet's disease has some role in the formation of cerebral aneurysms in some cases. In our patient, we were not certain at the first admission whether the aetiology of the aneurysm was related to Behçet's disease. However, the second aneurysm was found in association with the findings of vasculitis and simultaneously with the appearance of manifestations of the 
disease. These facts support the hypothesis that Behçet's disease is related to the aetiology of this aneurysm. Interestingly, the aneurysm disappeared after steroid therapy. This is the first report that shows the effectiveness of steroids in treating cerebral aneurysms associated with Behçet's disease, although spontaneous resolution of the aneurysm cannot be completely ruled out. In isolated CNS angiitis, Biller et al also described the possible resolution of one of multiple aneurysms after immunosuppressive therapy. ${ }^{3}$ However, Kerr et al described a case of a 12 year old boy with Behçet's disease, who had developed multiple aneurysms and subarachnoid haemorrhage in rapid succession even under steroid therapy. ${ }^{10}$ Also, several patients with systemic lupus erythematosus showed aneurysm rupture during the course of steroid therapy. ${ }^{2}$ In these patients, prolonged steroid therapy had been given before the diagnosis of cerebral aneurysms. Therefore, the disease might have become resistant to the steroid therapy. In conclusion, cerebral aneurysms may be associated with Behçet's disease. Surgical treatment is the first choice if they have already ruptured. However, steroid therapy may also be effective in patients with unruptured small aneurysms who have not received steroid therapy previously.
1 Sakaki T, Morimoto T, Utsumi S. Cerebral transmural angiitis and ruptured cerebral aneurysm in patients with systemic lupus erythematosus. Neurochirurgia 1990;33: $132-35$.

2 Kawamata T, Kagawa M, Kubo O, et al. Clinicopathological studies of three cases of cerebral aneurysms associated with systemic lupus erythematosus. Neurol Surg 1991;19:633639. (In Japanese.)

3 Biller J, Loftus CM, Moore SA, et al. Isolated central nervous system angiitis first presenting as spontaneous intracranial hemorrhage. Neurosurgery 1987;20:310-15.

4 Nishikawa M, Sakamoto H, Katsuyama J, et al. Multiple appearing vanishing aneurysms: primary angiitis of the central nervous system. f Neurosurg 1998;88:133-7.

5 Shimizu T, Ehrlich GE, Hayashi K. Behçet disease (Behçet syndrome). Semin Arthritis Rheum 1979;8:223-60.

6 Benamour S, Zeroual B, Bennis R, et al. Behçet disease. 316 Benamour S, Zeroual B, Bennis R, et al. Behçet disea
cases. Presse Méd 1990;19:1485-9. (In French.)

7 Al-Dalaan AN, Al Balaa SR, El Ramhi, et al. Behçet disease Al-Dalaan AN, Al Balaa SR, El Ramhi, et al. Be

8 Katoh K, Matsunaga K, Ishigatsubo Y, et al. Pathologically defined neuro-, vasculo-, entero-Behçet disease. F Rheumatol 1985;12:1186-90

9 Buge A, Rancurel VG, Dechy H, et al. Behçet disease with multiple intracranial aneurysms. Rev Neurol 1987;143:8325. (In French.)

10 Kerr JS, Roach ES, Sinal SH, et al. Intracranial arterial aneurysm complicating Behçet disease. $\mathcal{F}$ Child Neurol 1989;4:147-9

11 Tsuji S, Suzuki Y, Tomiii M, et al. Behçet disease associated with multiple cerebral aneurysms and downhill esophageal varices caused by superior vena cava obstruction: a case report. Ryumachi 1990;30:375-381.

12 Hassen-Kohdia R, Declemy S, Batt M, et al. Behcet's disease with multiple arterial lesions and voluminous
hemangioma of the brain. $\mathcal{F}$ Mal Vasc 1991;16:383-6. (In French.)

13 Ildan F, Göçer AI, Bagdatouglu H, et al. Intracranial aneurysm complicating Behçet disease. Neurosurg Rev 1996;19: 53-6

14 El Abbadi N, El Mostarchid B, Ababou A, et al. Behçet disease with multiple intracranial aneurysms: a case report. $\mathcal{f}$ Mal Vasc 1999;24:225-8. (In French.) 